\title{
Global, Regional, and National Burden of Kidney, Bladder, and Prostate Cancers and Their Attributable Risk Factors, 1990-2019
}

Hao Zi

Wuhan University Zhongnan Hospital

Xie-Yuan Leng

Anhui Medical University

Xiao-Feng Xu

Xi'an Jiaotong University

Qiao Huang

Wuhan University Zhongnan Hospital

Hong Weng

Wuhan University Zhongnan Hospital

Shao-Hua He

Wuhan University Zhongnan Hospital

Cong Zhu

Wuhan University Zhongnan Hospital

Lu-Yao Li

Wuhan University Zhongnan Hospital

Jia-Min Gu

Wuhan University Zhongnan Hospital

Xu-Hui Li

Wuhan University Zhongnan Hospital

Dao-Jing Ming

Wuhan University Zhongnan Hospital

Xiao-Dong Li

Henan University

Shuai Yuan

Wuhan University Zhongnan Hospital

Xing-Huan Wang

Wuhan University Zhongnan Hospital

Da-Lin He

Xi'an Jiaotong University

Xian-Tao Zeng ( $\nabla$ zengxiantao1128@163.com ) 


\section{Research}

Keywords: Genitourinary cancer, Kidney cancer, Bladder cancer, Prostate cancer, Incidence, Mortality, Disability-adjusted life-years, Burden of disease

Posted Date: May 5th, 2021

DOl: https://doi.org/10.21203/rs.3.rs-482279/v1

License: (9) This work is licensed under a Creative Commons Attribution 4.0 International License. Read Full License

Version of Record: A version of this preprint was published at Military Medical Research on November 24th, 2021. See the published version at https://doi.org/10.1186/s40779-021-00354-z. 
1 Global, regional, and national burden of kidney, bladder, and prostate cancers and their

$4 \quad$ Hao Zi ${ }^{1,2}$, Xie-Yuan Leng ${ }^{1,3}$, Xiao-Feng Xu ${ }^{4,5}$, Qiao Huang ${ }^{1}$, Hong Weng ${ }^{1,2}$, Shao-Hua He ${ }^{1,2,6}$,

5 Cong $\mathrm{Zhu}^{1,2}$, Lu-Yao Li ${ }^{1,7}$, Jia-Min Gu${ }^{1,2}$, Xu-Hui Li ${ }^{1}$, Dao-Jing Ming ${ }^{1,7}$, Xiao-Dong Li ${ }^{7,8}$, Shuai

6 Yuan ${ }^{1 *}$, Xing-Huan Wang ${ }^{1,2,9 *}$, Da-Lin He ${ }^{5 *}$, Xian-Tao Zeng ${ }^{1,2,9 *}$

8 1. Center for Evidence-Based and Translational Medicine, Zhongnan Hospital of Wuhan

9 University, Wuhan 430071, China;

2. Department of Urology, Zhongnan Hospital of Wuhan University, Wuhan 430071, China;

3. The First School of Clinical Medicine, Anhui Medical University, Hefei 230000, China;

4. Department of Urology, Xianyang Central Hospital, Xianyang 712000, China;

5. Department of Urology, The First Affiliated Hospital of Xi'an Jiaotong University, Xi'an 710061, China;

6. Precision Medicine Centre, the Second People's Hospital of Huaihua, Huaihua 418000, China;

7. Institutes of Evidence-based Medicine and Knowledge Translation, Henan University, Kaifeng, 475000, China;

8. Department of Urology, Huaihe Hospital of Henan University, Kaifeng 475000, China;

9. Institute of Urology, Wuhan University, Wuhan 430071, China.

*Corresponding author:

Shuai Yuan, E-mail: yuanshuai021@whu.edu.cn; 
Xing-Huan Wang, E-mail: wangxinghuan1965@163.com;

Da-Lin He, E-mail: hedl@xjtu.edu.cn;

Xian-Tao Zeng, E-mail: zengxiantao1128@whu.edu.cn, zengxiantao1128@163.com.

\section{Word count (abstract): 269}

\section{Abstract}

Background: To investigate the burden and attributable risk factors for three genitourinary cancers in 204 countries and territories during 30 years.

Methods: We extracted data of kidney, bladder, and prostate cancers from the Global Burden of Disease 2019 database, including incidence, mortality, disability-adjusted life-years (DALYs), and the attributable risk factors from 1990 to 2019. Estimated annual percentage changes (EAPC) were calculated to assess the changes in age-standardized incidence rate, age-standardized mortality rate (ASMR), and age-standardized DALYs rate (ASDR). The associations between cancers burden and socio-demographic index (SDI) were also analyzed.

Results: Compared with 1990, the global incident cases of kidney, bladder, and prostate cancers have increased by $154.78 \%, 123.34 \%$, and $169.11 \%$ in 2019 . The ASMR and ASDR of bladder cancer $(\mathrm{EAPC}=-0.68$ for $\mathrm{ASMR}, \mathrm{EAPC}=-0.83$ for $\mathrm{ASDR})$ and prostate cancer $(\mathrm{EAPC}=-0.75$ for ASMR, EAPC $=-0.71$ for ASDR) showed a downward trend, but kidney cancer increased (EAPC $=0.35$ for ASMR, EAPC $=0.12$ for ASDR). Of all cancers, incidence, mortality, and DALYs were higher in the high-level SDI regions and countries. The burden of bladder cancer and prostate cancer was mainly distributed among older men, while the burden of kidney cancer increases among 
younger men. Smoking related mortality and DALYs decreased, but high body-mass index and high fasting plasma glucose related mortality and DALYs increased among kidney, bladder, and prostate cancers between 1990 and 2019.

Conclusions: Kidney, bladder, and prostate cancers remain the major global public health challenge until 2019. We suggest the 204 countries and territories to take positive cancer prevention and intervention strategies, especially the urologists and urological related academic associations.

Key Words: Genitourinary cancer; Kidney cancer; Bladder cancer; Prostate cancer; Incidence; Mortality; Disability-adjusted life-years; Burden of disease

\section{Background}

Kidney, bladder, and prostate cancers are the most common genitourinary cancers, caused global $0.39,0.47$, and 1.33 million new cases in 2017 [1]. Previous studies have reported notable heterogeneity in the disease burden of genitourinary cancers at the geographic location, temporal trend, and socio-demographic index (SDI) levels [2-4]; however, none of them reported comprehensive data and reflected the changed spectrum. The incidence and mortality on genitourinary cancers have changed significantly in recent years, with the world population growth and aging, the life expectancy increased, and the socioeconomic development $[1,5,6]$. Hence, longterm, comprehensive, and accurate assessment of the disease burden is the necessary for health policy decision making.

The Global Burden of Disease (GBD) 2019 evaluated 369 diseases and injuries worldwide and compared their prevalence in different regions and countries, providing strategies for better 
improving population health and also providing valuable information for health policy development [7-9]. However, the information of kidney, bladder, and prostate cancers remain need to be further clarified. In this study, we used the data from the GBD 2019 to analyze the incidence, mortality, disability-adjusted life-years (DALYs) of these three cancers, and also analyzed their attributable risk factors from 1990 to 2019.

\section{Materials and Methods}

\section{Data source}

GBD 2019 is updated and expanded on the basis of GBD 2017 and provide the most up-to-date global health data. GBD 2019 includes data on mortality and morbidity in 204 countries and territories, 369 diseases and injuries, and 87 risk factors from 1990 to 2019. In this study, incidence, mortality, DALYs, and its corresponding age-standardized rates (ASR) by world standard population of kidney, bladder, and prostate cancers were gathered by the Global Health Data Exchange query tool (http://ghdx.healthdata.org/gbd-results-tool). Annual deaths and DALYs attributable to 87 risk factors are also available from the GBD results tool. The GBD world population standard was used for the calculation of ASR. The $95 \%$ uncertainty interval (UI) were reported for all estimates. The SDI is a composite index of development status strongly correlated with health outcomes, and the dataset are available online (http://ghdx.healthdata.org/gbd-2019). A region or country with an SDI of 0 would have a theoretical minimum level of development relevant to health, while a region or country with an SDI of 1 would have a theoretical maximum level. In addition, age group and sex were also extracted from GBD results tool to analyze the burden of kidney, bladder, and prostate cancers. 
90

91

92

93

\section{Cancer definition}

For GBD 2019, all cancers were defined by International Classification of Diseases (ICD) diagnostic criteria. The associated ICD-9 and ICD-10 codes of kidney, bladder, and prostate cancers for incidence and mortality data were as follows: kidney cancer (C64-C64.2, C64.9-C65.9, Z80.51, Z85.52-Z85.54, 189-189.1, 189.5-189.6, 209.24, C64-C65.9, D30.0-D30.1, D41.0-D41.1, and 223.0-223.1), bladder cancer (C67-C67.9, Z12.6-Z12.79, Z80.52, Z85.51, 188-188.9, V10.51, V16.52, V76.3, D09.0, D30.3, D41.4-D41.8, D49.4, 223.3, 233.7, 236.7, and 239.4), and prostate cancer (C61-C61.9, Z12.5, Z80.42, Z85.46, 185-185.9, V10.46, V16.42, V76.44, D07.5, D29.1, D40.0, 222.2, and 236.5). The incidence, mortality, and DALYs estimation for genitourinary cancers were described in the GBD 2019 study [7].

\section{Attributable risk factors}

The attributable risk factors for the kidney cancer related death and DALYs were smoking, high body-mass index, and occupational exposure to trichloroethylene. The attributable risk factors for the bladder cancer related death and DALYs were smoking and high fasting plasma glucose. The attributable risk factor for the prostate cancer related death and DALYs was smoking. The percentage of cancer related death and DALYs are available in GBD results tool. Details about the definitions of these risk factors and their relative risk for kidney, bladder, and prostate cancers were described elsewhere [8].

\section{Statistical analysis}


111 The general methodology and estimation process of GBD 2019 have been introduced in previous 112 publications [7]. The ASR (per 100,000 population) was calculated by the sum of the products of 113 age-specific rates $\left(a_{i}\right.$, where $i$ denotes the $i^{\text {th }}$ age) and the number of population (or weight $\left.w_{i}\right)$ in the 114 same age group $i$ of the selected reference standard population, divided by the sum of the standard 115 population weights: ASR $=\frac{\sum_{i=1}^{A} a_{i} w_{i}}{\sum_{i=1}^{A} w_{i}} \times 100,000$. We used estimated annual percentage changes (EAPC) 116 to describe the trend of ASR within a specified time interval. The natural logarithm of ASR is linear 117 with time. The EAPC were estimated by a linear regression model: $y=\alpha+\beta x+\varepsilon$, where $y$ is $\ln (\mathrm{ASR})$, $118 x$ is the calendar year, and $\varepsilon$ is the error term. The EAPC were calculated as $100 \times(\exp (\beta)-1)$ and its $95 \%$ confidence interval (CI) can be obtained from the linear regression model. When the estimated EAPC value and its lower 95\% CI were both $>0$, ASR is considered as an upward trend.

121 Conversely, if the estimated EAPC value and its upper 95\% CI were both $<0$, ASR is considered a downward trend. Data visualization and statistics were performed using R software (Version 4.0.5) and Microsoft Excel (Version 2016).

\section{Results}

\section{Global incidence, mortality and DALYs} In 2019, the global incident cases of kidney, bladder, and prostate cancers were 371,747 (95\% UI 344,594 to 402,350 ), 524,305 ( $95 \%$ UI 475,952 to 569,434 ) and $1,410,452$ ( $95 \%$ UI $1,227,900$ to $1,825,766)$ (Table 1). Compared with 1990, prostate cancer (169.11\%) had the largest increase, followed by kidney cancer (154.78\%) and bladder cancer (123.34\%). Between 1990 and 2019, prostate cancer had the highest percentage of incident cases among genitourinary cancers, followed by bladder and kidney cancers (Figure S1a). From 1990 to 2019, the age-standardized incidence 
133

134

135

136

137

rate (ASIR) of prostate cancer has been much higher than kidney and bladder cancers (Figure S2a). The EAPC of ASIR in genitourinary cancers showed an upward trend, except for female bladder cancer $(\mathrm{EAPC}=-0.24,95 \% \mathrm{CI}-0.29$ to -0.18$)$.

Worldwide, prostate cancer caused 486,836 male deaths in 2019, which was 4.48 times of kidney cancer and 2.88 times of bladder cancer. Compared with 1990, the percentage of deaths from prostate and kidney cancers increased, while the percentage of deaths from bladder cancer decreased in 2019 (Figure S1b). General, the age-standardized mortality rate (ASMR) for bladder cancer and prostate cancer showed a downward trend from 1990 to 2019 (Figure S2b). The ASMR for kidney cancer showed an upward trend before 2010, and has gradually declined in recent years. Over the past 30 years, the EAPC of ASMR showed an upward trend only in kidney cancer $(\mathrm{EAPC}=0.35$, 95\% CI 0.26-0.45).

Prostate cancer caused $8,644,870$ (95\% UI 7,548,021 to $10,559,866$ ) DALYs in 2019 , more than the sum of bladder and kidney cancers. The percentage of DALYs due to prostate and kidney cancers continues to expand from 1990 to 2019 (Figure S1c). The age-standardized DALYs rate (ASDR) of these three cancers had a consistent trend with ASMR in the past three decades (Figure S2c). The EAPC of ASDR had the most significant downward trend in female bladder cancer (EAPC $=-1.04$, $95 \%$ CI -1.08 to -0.99$)$.

\section{Regional incidence, mortality and DALYS}

In 2019, the regions had the most incident cases of prostate cancer were High-income North 
155

156

157

America (331,890, 95\% UI 262,386 to 494,583), Western Europe (325,494, 95\% UI 267,128 to 469,919), and East Asia (161,969, 95\% UI 126,342 to 213,686) (Table S1). It is worth noting that most of the new cases of bladder and kidney cancers were also distributed in these three regions. In addition, the ASIR of bladder and kidney cancers showed the most significant upward trend in East Asia, while the ASIR of prostate cancer showed a downward trend only in High-income North America (Figure 1).

Western Europe had the most deaths of prostate (95,769, 95\% UI 79,228 to 133,047), bladder $(50,511,95 \%$ UI 45,163 to 54,465$)$ and kidney cancers $(34,359,95 \%$ UI 31,508 to 36,302$)$ in 2019 (Table S2). The highest ASMR of kidney, bladder, and prostate cancers were Southern Latin America (5.05, 95\% UI 4.59 to 5.51), Central Europe (5.29, 95\% UI 4.66 to 5.99), and Southern Sub-Saharan Africa (45.08, 95\% UI 34.35 to 50.53). Stark regional differences were observed in ASMR of genitourinary cancers during the past 30 years (Figure S3). For example, the fastest increase of ASMR in kidney cancer were observed in East Asia, followed by Central Europe, North Africa and Middle East, and Eastern Sub-Saharan Africa.

The highest DALYs of kidney and bladder cancers were observed in East Asia, and prostate cancer were observed in High-income North America in 2019 (Table S3). The ASDR of kidney, bladder, and prostate cancers were highest in Southern Latin America (124.32, 95\% UI 113.32 to 135.49), Central Europe (107.36, 95\% UI 93.95 to 122.07), and Southern Sub-Saharan Africa (726.31, 95\% UI 574.70 to 822.50). From 1990 to 2019, East Asia, Oceania, and Western Sub-Saharan Africa had the fastest increase in ASDR for kidney, bladder and prostate cancers, respectively (Figure S4). 
National incidence, mortality and DALYs

179

180

181

182

183

184

185

186

187

188

189

190

Among 204 countries and territories, USA (308,584, 95\% UI 240,778 to 460,979), China (153,448, $95 \%$ UI 118,400 to 204,943$)$, and Germany $(75,380,95 \%$ UI 53,645 to 104,000$)$ had the most prostate cancer incident cases in 2019 (Table S4). The most incident cases of bladder cancer and kidney cancer were found in China $(100,020,95 \%$ UI 83,242 to 118,654$)$ and USA $(61,541,95 \%$ UI 52,477 to 71,382). The ASIR of kidney, bladder, and prostate cancers were highest in Czechia (15.66, 95\% UI 12.62 to 19.55$)$, Monaco (31.92, $95 \%$ UI 23.32 to 56.95$)$, and Saint Kitts and Nevis (235.26, 95\% UI 184.24 to 287.38). From 1990 to 2019 , the Bulgaria (EAPC $=6.24,95 \%$ CI 5.32 to 7.16$)$, Cabo Verde (EAPC $=3.88,95 \%$ CI 3.19 to 4.57$)$, and Estonia $(\mathrm{EAPC}=4.31,95 \% \mathrm{CI} 3.94$ to 4.69) had the most significant increase in the ASIR of kidney, bladder, and prostate cancers, respectively (Figure 2).

In 2019, China had the most deaths of kidney, bladder, and prostate cancers, followed by USA and Russian Federation (Table S4). The ASMR of kidney cancer was highest in Uruguay (6.56, 95\% UI 5.90 to 7.23$)$, and lowest in Papua New Guinea (0.49, $95 \%$ UI 0.34 to 0.75$)$. Lebanon (10.38, 95\% UI 8.08 to 13.72$)$ and Dominica $(126.28,95 \%$ UI 93.14 to 156.53$)$ showed the highest ASMR of bladder cancer and prostate cancer. The EAPC of ASMR showed that kidney, bladder, and prostate cancers had the most significant increase in Bulgaria (EAPC $=5.83,95 \%$ CI 4.92 to 6.75), Northern Mariana Islands $(\mathrm{EAPC}=3.22,95 \%$ CI 2.62 to 3.84$)$, and Cabo Verde $(\mathrm{EAPC}=2.53,95 \%$ CI 1.74 to 3.33), respectively (Figure S5). 
The most DALYs caused by kidney, bladder, and prostate cancers were also found among China and USA in 2019 (Table S4). The number of DALYs for kidney, bladder, and prostate cancers in China was 1.5 times, 2.1 times, and 1.1 times higher than USA, respectively. The highest ASDR for kidney, bladder, and prostate cancers was Uruguay (166.63, 95\% UI 149.92 to 184.54), Egypt (201.75, 95\% UI 132.14 to 294.39), and Dominica (1,923.95, 95\% UI 1,428.24 to 2,394.31). The Bulgaria $(\mathrm{EAPC}=5.71,95 \% \mathrm{CI} 4.80$ to 6.62$)$, Northern Mariana Islands $(\mathrm{EAPC}=3.37,95 \% \mathrm{CI}$ 2.73 to 4.02 ), and Georgia ( $\mathrm{EAPC}=2.67,95 \% \mathrm{CI} 2.07$ to 3.27 ) were the most significant increase in ASDR for kidney, bladder, and prostate cancers from 1990 to 2019, respectively (Figure S6).

\section{Burden of genitourinary cancers by SDI}

High-level SDI regions had higher ASIR of kidney cancer in global and 21 regions (Figure 3a).

Similarly, the ASMR and ASDR of kidney cancer in high-level SDI regions were higher than the low-level SDI regions (Figure S7-8). Among the 204 countries and territories, the ASIR of kidney cancer in Czechia were much higher than the expected levels in 2019 (Figure 3b). The associations between ASIR and SDI were also found among bladder and prostate cancers at the regional and national level (Figure S9-10). However, no associations between ASMR, ASDR and SDI were observed among bladder and prostate cancers (Figure S11-14).

\section{Burden of genitourinary cancers by age and sex}

The 65-69 years age group had the most incident cases of kidney cancer in 2019 (Figure 4a). The incidence rate of kidney cancer was highest in 85-89 years age group among men (47.97, 95\% UI 41.44 to 52.79$)$ and $90-94$ years age group among women (23.06, 95\% UI 17.53 to 26.68$)$. The 
221

222

223

224

225

highest mortality rate of kidney cancer was observed in the oldest age groups. The incident cases of bladder cancer increase with age, peaking in 70-74 years old, and then decreases (Figure 4d). In the 95 years or older age group, the incidence rate, mortality rate, and DALYs rate of bladder cancer in men were 3.4 times, 3.3 times, and 3.3 times higher than women, respectively. The incident cases of prostate cancer started climbing at 55-59 years old, reached a peak at 70-74 years old, and dropped in the oldest age groups (Figure 4g).

\section{Attributable risk factors}

Between 1990 and 2019, the percentage of kidney cancer DALYs were attributable to the smoking dropped from $18.19 \%$ to $16.95 \%$, while high body-mass index rose from $13.98 \%$ to $18.55 \%$ (Table S5). Smoking and high fasting plasma glucose were mainly attributable risk factors of bladder cancer death and DALYs. In 2019, 6.01\% of deaths and $6.6 \%$ of DALYs caused by prostate cancer were attributable to smoking.

\section{Discussion}

This study revealed that the global, regional, and national burden of genitourinary cancers and their attributable risk factors have changed in the past 30 years. Compared with 1990, the proportion of prostate cancer new cases expanded and still accounted for the majority among these cancers, the proportion of bladder cancer decreased and ranked 2th among them, and the proportion of kidney cancer remained stable in 2019. We observed the significant increase in ASIR for kidney cancer $(\mathrm{EAPC}=0.87)$, followed by prostate cancer $(\mathrm{EAPC}=0.26)$ and bladder cancer $(\mathrm{EAPC}=0.07)$ at the global level. From these changes, we found the kidney cancer still represent a small percentage 
243

244

of genitourinary cancers, compared with bladder and prostate cancers, even though kidney cancer has the fastest increase in ASIR. The changed global incidence of genitourinary cancers indicated the evolution of early detection of disease, such as prostate-specific antigen screening for prostate cancer and cross-sectional imaging for kidney cancer [10,11]. For example, the widespread use of routine prostate-specific antigen testing led to a rapid increase in the incidence of prostate cancer in the early 1990's among USA; however, prostate cancer incidence has been declining in recent years with the reduction in routine prostate-specific antigen testing [12-14]. Likewise, advances in imaging technology have led to an increase in the detection rate of kidney cancer in developed countries [10]. Other reasons may partly explain the accelerated incidence of genitourinary cancers, included aging population and changed lifestyles. Besides, deaths caused by genitourinary cancers were increased but fortunately we see the opposite trend in the ASMR, especially for prostate and bladder cancers. This trend was also showed in DALYs and ASDR among these three genitourinary cancers. Therefore, the decline in mortality and DALYs proves the effectiveness of current cancer prevention and treatment strategies. In the future, the global epidemiological studies of cancer, such as the GBD program and the Global Cancer Observatory project [15], should continue to provide evidence to guide decision-making.

The variation in incidence, mortality, and DALYs of genitourinary cancers was noted at the regional and national level. In the past three decades, the incidence of bladder cancer has shown a downward trend in 8 regions, while prostate cancer and kidney cancer has shown an upward trend almost in 21 regions. This results of these three cancers were consistent with the previous studies $[2,4,16]$. The prevalence of risk factors associated with genitourinary cancer and the spread of early detection 
could partially explain the incidence trend in these regions. Mortality and DALYs of genitourinary cancers showed significant decrease in developed regions such as Australasia and High-income North America. Better health care policies and disease prevention awareness may play an important role in reducing the burden of genitourinary cancers. Moreover, SDI was associated with the incidence, mortality, and DALYs of genitourinary cancers. For all cancer, high-level SDI regions and countries have higher incidence, mortality and DALYs. However, we have noticed that the mortality and DALYs of genitourinary cancers in high-level SDI regions and countries showed a steady or downward trend. This downward trend was most obvious in prostate cancer, followed by bladder cancer and kidney cancer. The appropriate cancer prevention and intervention strategies is needed in different social development degree countries.

The incidence and burden of genitourinary cancers were significantly higher among the elderly men. Previous studies based on the GBD 2017 data have also shown that the burden of genitourinary cancers in the elderly was more serious $[2-4,17,18]$. The aging population and the increase in life expectancy are one of the factors that affect the burden of disease, but cancer-related risk factors also play an important role [19-23]. Smoking is a shared risk factor for these three cancers and shows a decreasing contribution to cancer death and DALYs in the past 30 years. Notably, the burden of disease associated with high fasting plasma glucose and high body-mass index is increasing. This shows the effectiveness of previous tobacco control strategies, but also suggests the necessary to explore new preventive measures to address the increased metabolic risk factors. In addition, there are excellent international health programs that are worth learning and replicating [24]. For example, the Universal Health Coverage program led by the WHO enables everyone to access the health 
287

288

services and ensures that the quality of those services is good enough to improve the health of the people who receive them. As such, urologists and urological related academic associations could develop standards and tools to provide guidance for the implementation of cancer prevention, screening and treatment.

Today and in the near future, the epidemiological studies of genitourinary cancers under the COVID-19 pandemic will face the unprecedented challenges. First, epidemiological data of genitourinary cancers may not be as accurate as in the past. A global survey showed COVID-19 had led to significant delay in outpatient care and surgery in urology, with an average delay of more than 8 weeks [25]. Delays in the normal medical order, which may result in a large number of patients not being accounted for the morbidity and mortality. Second, COVID-19 may increase the disease burden of genitourinary cancers. Joint effects of COVID-19 and cancer sequelae may be seen in DALYs, including physical and mental health [26]. Finally, the ongoing effects of the pandemic may not end in a short time. Hence, to divide the death cause of died genitourinary cancers patients who also undergo COVID-19 may help to accurately assess the disease burden.

Although our research was based on GBD 2019 data and methods, it still had some limitations. First, the accuracy of the GBD data depends on the quality of the existing data in each country, we believe the quality was different among countries with different social, economic and cultural backgrounds; hence, the results of smaller countries or countries with low levels of development should be interpreted with caution. Second, differences in reporting approaches and case definitions may cause information to be missed. We suggest the WHO perform a unified design methodology and 
309

310

311

312

313

314

315

316

317

318

319

320

321

322

323

324

325

326

327

328

329

330

procedure to collect data. Finally, although there are available data on genitourinary cancers in GBD

2019, it does not include all risk factors and etiologies, so the disease burden cannot be fully assessed.

\section{Conclusions}

In conclusion, kidney, bladder, and prostate cancers remain the major global public health challenge until 2019. We suggest the 204 countries and territories to take positive cancer prevention and intervention strategies, especially the urologists and urological related academic associations.

\section{Abbreviations}

ASDR: age-standardized DALYs rate

ASIR: age-standardized incidence rate

ASMR: age-standardized mortality rate

ASR: age-standardized rates

CI: confidence interval

DALYs: disability-adjusted life-years

EAPC: estimated annual percentage changes

GBD: Global Burden of Disease

ICD: International Classification of Diseases

SDI: socio-demographic index

UI: uncertainty interval

\section{Declarations}


Not applicable.

\section{Consent for publication}

335

Not applicable.

\section{Availability of data and materials}

The datasets generated during the current study are available in the Global Health Data Exchange query tool (http://ghdx.healthdata.org/gbd-results-tool).

\section{Competing interests}

The authors declare that they have no competing interests.

343

344

345

346

347 SY, XHW, DLH, and XTZ. All authors read and approved the final manuscript. 
353

354

355

356

357

358

359

360

361

362

363

364

365

366

367

368

369

370

371

372

373

374

\section{Acknowledgements}

Not applicable.

\section{References}

1. Fitzmaurice C, Abate D, Abbasi N, Abbastabar H, Abd-Allah F, Abdel-Rahman O, et al. Global, Regional, and National Cancer Incidence, Mortality, Years of Life Lost, Years Lived With Disability, and Disability-Adjusted Life-Years for 29 Cancer Groups, 1990 to 2017: A Systematic Analysis for the Global Burden of Disease Study. JAMA Oncol. 2019;5(12):1749-68.

2. Zhai Z, Zheng Y, Li N, Deng Y, Zhou L, Tian T, et al. Incidence and disease burden of prostate cancer from 1990 to 2017: Results from the Global Burden of Disease Study 2017. Cancer. 2020;126(9):1969-78.

3. He H, Xie H, Chen Y, Li C, Han D, Xu F, et al. Global, regional, and national burdens of bladder cancer in 2017: estimates from the 2017 global burden of disease study. BMC Public Health. 2020;20(1):1693.

4. Bai X, Yi M, Dong B, Zheng X, Wu K. The global, regional, and national burden of kidney cancer and attributable risk factor analysis from 1990 to 2017. Exp Hematol Oncol. 2020;9:27.

5. Siegel RL, Miller KD, Jemal A. Cancer statistics, 2019. CA Cancer J Clin. 2019;69(1):7-34.

6. Zhang S, Sun K, Zheng R, Zeng H, Wang S, Chen R, et al. Cancer incidence and mortality in China, 2015. Journal of the National Cancer Center. 2020.

7. GBD 2019 Diseases and Injuries Collaborators. Global burden of 369 diseases and injuries in 204 countries and territories, 1990-2019: a systematic analysis for the Global Burden of Disease Study 2019. Lancet. 2020;396(10258):1204-22. 
375 8. GBD 2019 Risk Factors Collaborators. Global burden of 87 risk factors in 204 countries and territories, 1990-2019: a systematic analysis for the Global Burden of Disease Study 2019. Lancet. 2020;396(10258):1223-49.

9. GBD 2019 Demographics Collaborators. Global age-sex-specific fertility, mortality, healthy life expectancy (HALE), and population estimates in 204 countries and territories, 1950-2019: a comprehensive demographic analysis for the Global Burden of Disease Study 2019. Lancet.

10. Hock LM, Lynch J, Balaji KC. Increasing incidence of all stages of kidney cancer in the last 2 decades in the United States: an analysis of surveillance, epidemiology and end results program data. J Urol. 2002;167(1):57-60.

11. Fedewa SA, Ward EM, Brawley O, Jemal A. Recent Patterns of Prostate-Specific Antigen Testing for Prostate Cancer Screening in the United States. JAMA Internal Medicine. 2017;177(7):1040-2.

12. Goldenberg MG, Skeldon SC, Nayan M, Suppiah Y, Chow L, Fryml E, et al. Prostate-specific

14. Fleshner K, Carlsson SV, Roobol MJ. The effect of the USPSTF PSA screening recommendation 

J Urol. 2019;201(5):893-901.

17. Zi H, Peng M, Wang J, Hu H, Yang L, Yao H, et al. [An analysis of disease burden of bladder cancer in China from 1990 to 2017]. Yixue Xinzhi Zazhi. 2020;30(5):328-35.[Article in Chinese].

401

18. Deng T, Cai L, Chen Z, Guo J, Huang X, Ke C, et al. [Analysis of the burden of prostate cancer in China in 1990 and 2017]. Yixue Xinzhi Zazhi. 2020;30(4):252-9.[Article in Chinese].

19. Cumberbatch MG, Rota M, Catto JW, La Vecchia C. The Role of Tobacco Smoke in Bladder and Kidney Carcinogenesis: A Comparison of Exposures and Meta-analysis of Incidence and Mortality Risks. Eur Urol. 2016;70(3):458-66.

20. Colt JS, Friesen MC, Stewart PA, Donguk P, Johnson A, Schwenn M, et al. A case-control study of occupational exposure to metalworking fluids and bladder cancer risk among men. Occup Environ Med. 2014;71(10):667-74.

21. Pesch B, Taeger D, Johnen G, Gawrych K, Bonberg N, Schwentner C, et al. Screening for bladder cancer with urinary tumor markers in chemical workers with exposure to aromatic amines. Int Arch Occup Environ Health. 2014;87(7):715-24.

22. Capitanio U, Bensalah K, Bex A, Boorjian SA, Bray F, Coleman J, et al. Epidemiology of Renal Cell Carcinoma. Eur Urol. 2019;75(1):74-84.

23. Tan C, Tang CZ, Chen XS, Luo YJ. Association between medical resources and the proportion of oldest-old in the Chinese population. Mil Med Res. 2021;8(1):14.

24. Zakumumpa H, Bennett S, Ssengooba F. Leveraging the lessons learned from financing HIV programs to advance the universal health coverage (UHC) agenda in the East African Community. Glob Health Res Policy. 2019;4:27. 
25. Teoh JY, Ong WLK, Gonzalez-Padilla D, Castellani D, Dubin JM, Esperto F, et al. A Global Survey on the Impact of COVID-19 on Urological Services. Eur Urol. 2020;78(2):265-75.

26. Wallis CJD, Catto JWF, Finelli A, Glaser AW, Gore JL, Loeb S, et al. The Impact of the COVID19 Pandemic on Genitourinary Cancer Care: Re-envisioning the Future. Eur Urol. 2020;78(5):73142.

\section{Table}

Table 1. Global incidence, mortality and DALYs of genitourinary cancers from 1990 to 2019

\section{Figure Legends}

Figure 1. The EAPC of ASIR for genitourinary cancers in global and 21 regions. a, kidney cancer;

b, bladder cancer; c, prostate cancer. ASIR, age-standardized incidence rate; EAPC, estimated annual percentage change.

Figure 2. The EAPC of ASIR for genitourinary cancers in 204 countries and territories. a, kidney cancer; b, bladder cancer; c, prostate cancer. ASIR, age-standardized incidence rate; EAPC, estimated annual percentage change.

Figure 3. ASIR of kidney cancer for 21 regions and 204 countries and territories by SDI. a, 21 regions; b, 204 countries and territories. ASIR, age-standardized incidence rate; SDI: sociodemographic index.

Figure 4. Global incidence, mortality, and DALYs of genitourinary cancers by age and sex in 2019. $\mathbf{a}, \mathbf{b}$, and $\mathbf{c}$, incidence, mortality, and DALYs of kidney cancer; $\mathbf{d}, \mathbf{e}$, and $\mathbf{f}$, incidence, mortality, and 
441

442

443

444

445

446

447

448

449

450

451

452

453

454

455

456

457

DALYs of bladder cancer; $\mathbf{g}, \mathbf{h}$, and $\mathbf{i}$, incidence, mortality, and DALYs of prostate cancer. DALYs.

DALYs, disability-adjusted life-years.

\section{Supplementary material}

\section{Tables}

Table S1. Regional incident cases and age-standardized incidence rate of genitourinary cancers in 2019.

Table S2. Regional deaths and age-standardized mortality rate of genitourinary cancers in 2019.

Table S3. Regional DALYs and age-standardized DALYs rate of genitourinary cancers in 2019.

Table S4. The incidence, mortality and DALYs of genitourinary cancers among the top three and bottom three countries in 2019 .

Table S5. The percentage of genitourinary cancers deaths and DALYs attributable to risk factors in 1990 and 2019.

\section{Figure Legends}

Figure S1. The percentage of incident cases, deaths, and DALYs for genitourinary cancers in 1990 and 2019. a, incident cases; b, deaths; c, DALYs. DALYs, disability-adjusted life-years.

Figure S2. The global ASIR, ASMR, and ASDR of genitourinary cancers from 1990 to 2019. a, ASIR; b, ASMR; c, ASDR. ASIR, age-standardized incidence rate; ASMR, age-standardized mortality rate; ASDR, age-standardized DALYs rate; DALYs, disability-adjusted life-years.

Figure S3. The EAPC of ASMR for genitourinary cancers in global and 21 regions. ASMR, agestandardized mortality rate; EAPC, estimated annual percentage change. 
Figure S4. The EAPC of ASDR for genitourinary cancers in global and 21 regions. ASDR, agestandardized DALYs rate; EAPC, estimated annual percentage change.

Figure S5. The EAPC of ASMR for genitourinary cancers in 204 countries and territories. a, kidney cancer; b, bladder cancer; c, prostate cancer. ASMR, age-standardized mortality rate; EAPC, estimated annual percentage change.

Figure S6. The EAPC of ASDR for genitourinary cancers in 204 countries and territories. a, kidney cancer; b, bladder cancer; c, prostate cancer. ASDR, age-standardized DALYs rate; EAPC, estimated annual percentage change.

Figure S7. ASMR of kidney cancer for 21 regions and 204 countries and territories by SDI. a, 21 regions; b, 204 countries and territories. ASMR, age-standardized mortality rate; SDI: sociodemographic index.

Figure S8. ASDR of kidney cancer for 21 regions and 204 countries and territories by SDI. a, 21 regions; b, 204 countries and territories. ASDR, age-standardized DALYs rate; SDI: sociodemographic index.

Figure S9. ASIR of bladder cancer for 21 regions and 204 countries and territories by SDI. a, 21 regions; b, 204 countries and territories. ASIR, age-standardized incidence rate; SDI: sociodemographic index.

Figure S10. ASIR of prostate cancer for 21 regions and 204 countries and territories by SDI. a, 21 regions; b, 204 countries and territories. ASIR, age-standardized incidence rate; SDI: sociodemographic index.

Figure S11. ASMR of bladder cancer for 21 regions and 204 countries and territories by SDI. a, 21 regions; b, 204 countries and territories. ASMR, age-standardized mortality rate; SDI: 
sociodemographic index.

486 Figure S12. ASDR of bladder cancer for 21 regions and 204 countries and territories by SDI. a, 21

487 regions; b, 204 countries and territories. ASDR, age-standardized DALYs rate; SDI: 488 sociodemographic index.

489 Figure S13. ASMR of prostate cancer for 21 regions and 204 countries and territories by SDI. a, 49021 regions; b, 204 countries and territories. ASMR, age-standardized mortality rate; SDI: 491 sociodemographic index.

492 Figure S14. ASDR of prostate cancer for 21 regions and 204 countries and territories by SDI. a, 21 493 regions; b, 204 countries and territories. ASDR, age-standardized DALYs rate; SDI: 494 sociodemographic index. 
Figures
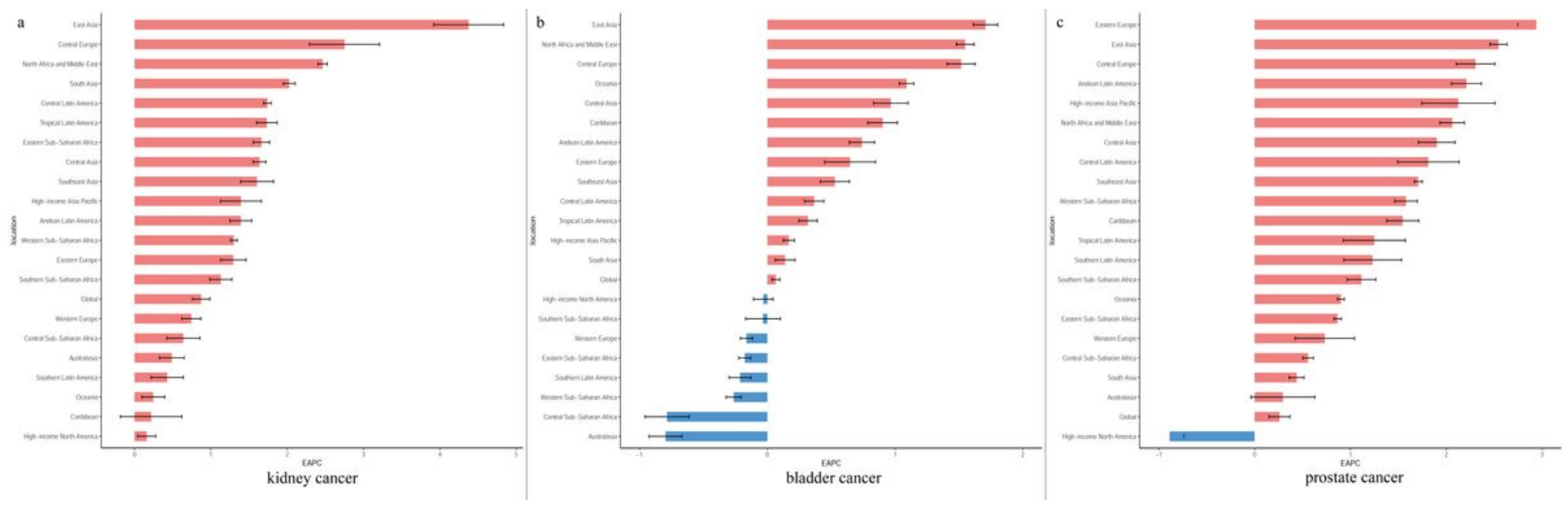

\section{Figure 1}

The EAPC of ASIR for genitourinary cancers in global and 21 regions. a, kidney cancer; b, bladder cancer; c, prostate cancer. ASIR, age-standardized incidence rate; EAPC, estimated annual percentage change. 

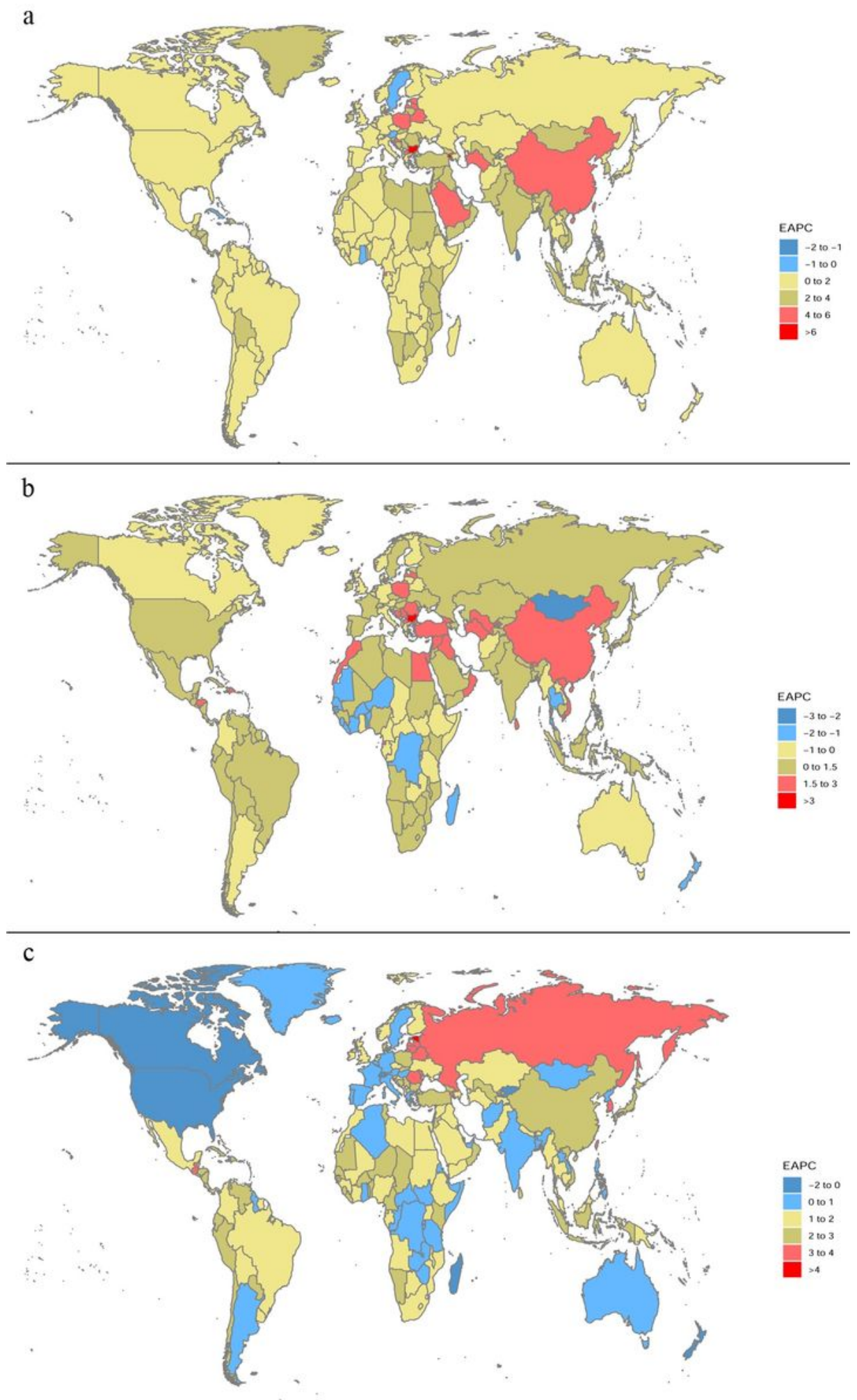

\section{Figure 2}

The EAPC of ASIR for genitourinary cancers in 204 countries and territories. a, kidney cancer; b, bladder cancer; c, prostate cancer. ASIR, age-standardized incidence rate; EAPC, estimated annual percentage change. Note: The designations employed and the presentation of the material on this map do not imply the expression of any opinion whatsoever on the part of Research Square concerning the legal status of 
any country, territory, city or area or of its authorities, or concerning the delimitation of its frontiers or boundaries. This map has been provided by the authors.
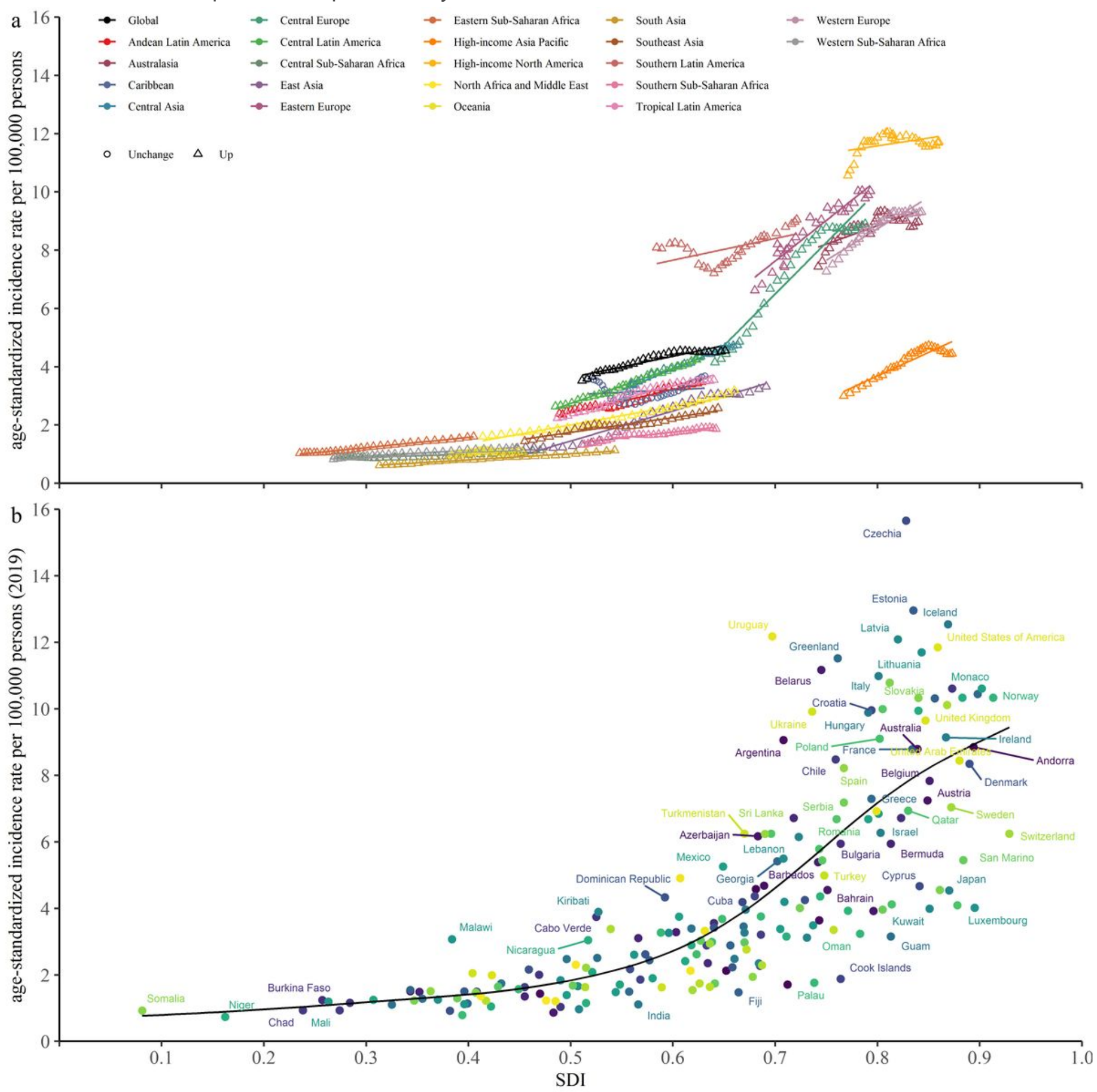

Figure 3

ASIR of kidney cancer for 21 regions and 204 countries and territories by SDI. a, 21 regions; b, 204 countries and territories. ASIR, age-standardized incidence rate; SDI: sociodemographic index. 

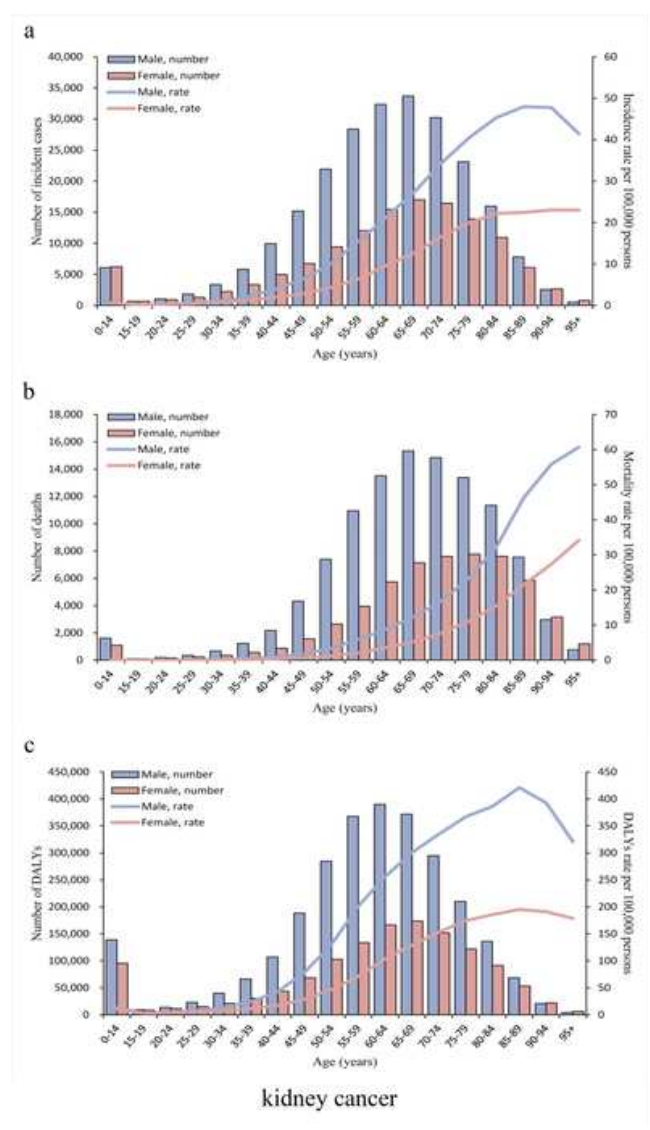
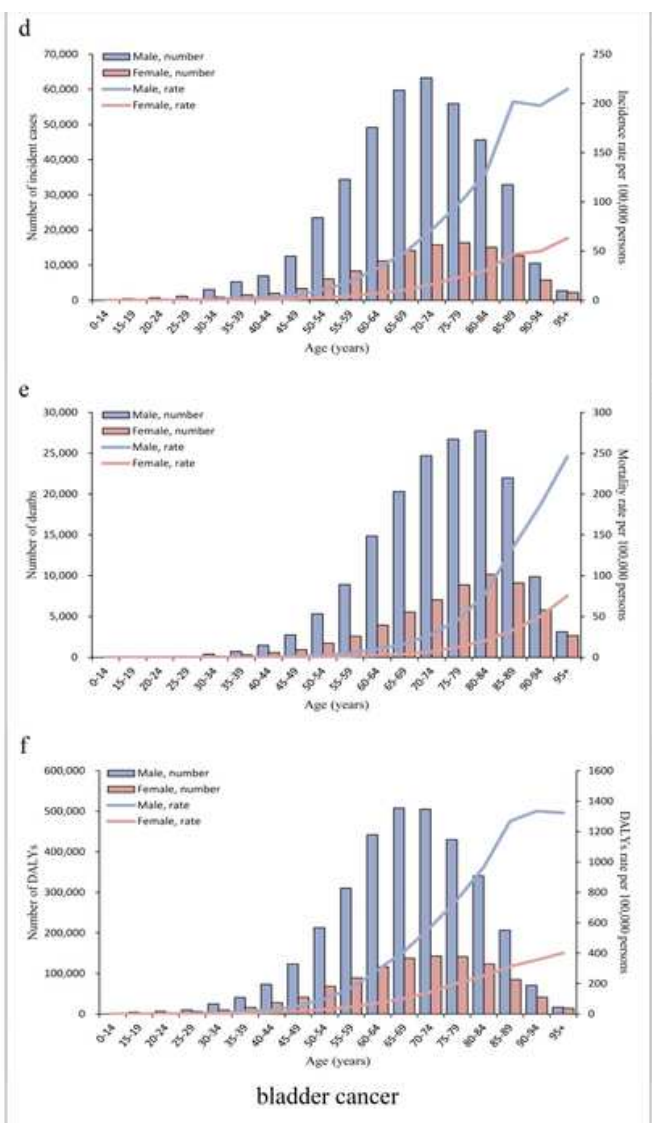

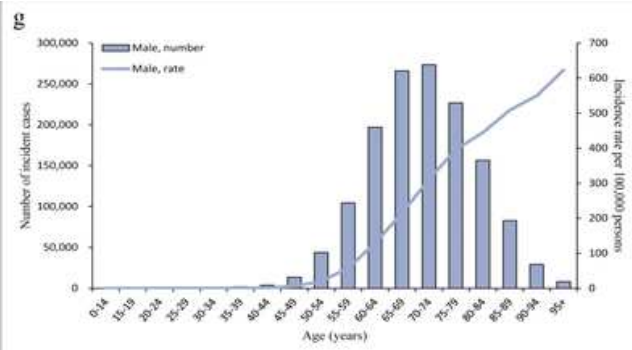

h
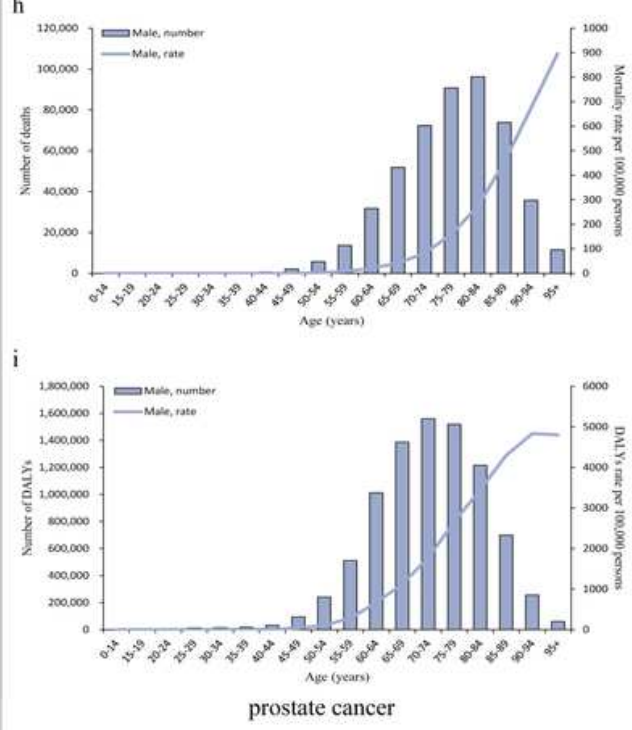

\section{Figure 4}

Global incidence, mortality, and DALYs of genitourinary cancers by age and sex in 2019. a, b, and c, incidence, mortality, and DALYs of kidney cancer; $d, e$, and $f$, incidence, mortality, and DALYs of bladder cancer; $\mathrm{g}$, h, and i, incidence, mortality, and DALYs of prostate cancer. DALYs. DALYs, disability-adjusted life-years.

\section{Supplementary Files}

This is a list of supplementary files associated with this preprint. Click to download.

- FigureS1.tif

- FigureS10.tif

- FigureS11.tif

- FigureS12.tif

- FigureS13.tif

- FigureS14.tif

- Figures2.tif

- Figures3.tif

- Figures4.tif 
- FigureS5.tif

- Figures6.tif

- Figures7.tif

- Figures8.tif

- Figures9.tif

- TableS1.docx

- TableS2.docx

- TableS3.docx

- Tables4.docx

- TableS5.docx 